\title{
Control of underactuated planar pronking through an embedded spring-mass Hopper template
}

\author{
M. Mert Ankaralı • Uluc̣ Saranlı
}

Received: 2 November 2009 / Accepted: 16 December 2010 / Published online: 29 December 2010

(C) Springer Science+Business Media, LLC 2010

\begin{abstract}
Autonomous use of legged robots in unstructured, outdoor settings requires dynamically dexterous behaviors to achieve sufficient speed and agility without overly complex and fragile mechanics and actuation. Among such behaviors is the relatively under-studied pronking (aka. stotting), a dynamic gait in which all legs are used in synchrony, usually resulting in relatively slow speeds but long flight phases and large jumping heights. Instantiations of this gait for robotic systems have been mostly limited to open-loop strategies, suffering from severe pitch instability for underactuated designs due to the lack of active feedback. However, both the kinematic simplicity of this gait and its dynamic nature suggest that the Spring-Loaded Inverted Pendulum model (SLIP) would be a good basis for the implementation of a more robust feedback controller for pronking. In this paper, we describe how template-based control, a controller structure based on the embedding of a simple dynamical "template" within a more complex "anchor" system, can be used to achieve very stable pronking for a planar, underactuated hexapod robot. In this context, high-level control of the gait is regulated through speed and height commands to the SLIP template, while the embedding controller ensures the stability of the remaining degrees of freedom. We use simulation studies to show that unlike existing open-loop alternatives, the resulting control struc-
\end{abstract}

M.M. Ankaralı

Dept. of Electrical and Electronics Eng., Middle East Technical University, Ankara, Turkey

e-mail: e134441@metu.edu.tr

U. Saranlı $(\bowtie)$

Dept. of Computer Engineering, Bilkent University, Ankara,

Turkey

e-mail: saranli@cs.bilkent.edu.tr ture provides explicit gait control authority and significant robustness against sensor and actuator noise.

Keywords Legged robots · Pronking · Inverse dynamics · Template based control · Dynamically dexterous locomotion $\cdot$ RHex $\cdot$ Hexapod robots

\section{Introduction}

\subsection{Motivation and background}

Legged robot morphologies admit a wider range of behavioral alternatives than more traditional tracked or wheeled platforms with added mobility provided by otherwise infeasible behaviors such as running (Altendorfer 2000), leaping and self-righting (Saranli et al. 2004). On the other hand, legged systems often suffer both from additional hardware complexity to support leg mechanisms, as well as increased difficulty in designing controllers that can robustly realize desired behaviors. One of the ways in which this mechanical complexity can be decreased is the use of dynamic modes of locomotion, wherein second order dynamics are properly designed, tuned and exploited to achieve a wide variety of behaviors even in the absence of full actuation (Allen et al. 2003; Sato and Buehler 2004; Saranli et al. 2004). Early instantiations of this idea can be found in Raibert's runners (Raibert 1986), capable of fast and stable locomotion on flat ground as well as dynamic maneuvers over obstacles. In practice, this approach also has the advantage of significantly improving robustness and decreasing power requirements as a result of using fewer actuators and the associated reduction in weight and complexity (Saranli et al. 2001). Unfortunately, the design, analysis and control of such dynamically dexterous legged platforms is more challenging 
Fig. 1 (Left) Snapshot of a planar hexapedal pronking stride, (right) the SLIP template

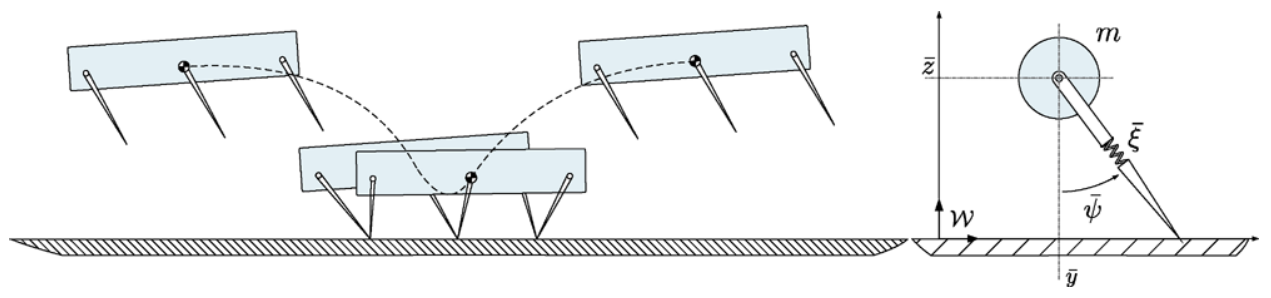

than simpler but slow, statically stable platforms due to difficulties in understanding and controlling second order dynamics. Despite substantial research in this domain, sufficiently general solutions to this problem remain elusive.

In this paper, we present the mathematical basis and a practical implementation of template based control of dynamic legged locomotion, a decompositional approach to isolate and independently control robot degrees of freedom that are relevant to the desired task (Full and Koditschek 1999). We concentrate on the pronking behavior for the hexapedal RHex platform (Saranli et al. 2001), whose robust and consistent realization in the absence of radial leg actuation has previously not been possible (McMordie 2002). Pronking is a gait adopted by legged animals wherein all legs are used in synchrony and a substantial flight phase is induced (see Fig. 1). This gait is often used by animals to signal their strength to potential predators (FitzGibbon and Fanshawe 1988; Caro 1994). Even though such a goal is unnecessary for robotic platforms, large jumping heights associated with this gait are potentially useful for locomotion on cluttered natural environments and may even increase efficiency by decreasing damping losses with shorter stance and longer flight phases. Moreover, the lateral symmetry of the gait admits the use of simpler, planar models and provides a rich domain for studying feedback control of dynamic legged locomotion, particularly in the presence of underactuated leg structures. Such a planar simplification also allows the analysis of similar gaits such as the trot and the pace (Berkemeier and Sukthankar 2005).

Due to sensory limitations of our experimental platform, we use a non-dimensional, previously validated planar simulation to provide a careful and thorough characterization of the stability properties and noise performance of the proposed pronking controller. The present paper extends on our previous results for alternating tripod running (Saranli 2002; Saranli and Koditschek 2003) to dynamic pronking, while also providing a more careful characterization of its stability properties and robustness against model and measurement uncertainty.

\subsection{Existing work}

There has been very little explicit focus on robotic pronking in the literature (McMordie 2002; Berkemeier and Sukthankar 2005; Chatzkos and Papadopoulos 2009), as opposed to the much more widely studied bounding behavior
(Raibert 1990; Poulakakis et al. 2005; Zou and Schmiedeler 2006; Chatzakos and Papadopoulos 2009). Existing control strategies for both types of behavior largely rely on simple open-loop strategies (e.g. with constant hip torque inputs or open-loop leg angle profiles) that offer little or no control authority over high level gait parameters and require extensive tuning to be successful. Even though the use of optimization methods promises to yield some insight into useful design criteria for robots capable of such highly dynamic behaviors (Chatzakos and Papadopoulos 2007), the range of operation and extensibility of resulting controllers remains limited.

In this context, there is significant biological (Duysens and de Crommert 1998; Kopell 2000) and engineering (Kuo 2002; Klavins et al. 2002) evidence to support the adoption of predominantly open-loop controllers with properly tuned passive dynamics and minimal feedback for reliable locomotion. Nevertheless, high-bandwidth feedback controllers based on accurate dynamic models of such systems are still necessary for the insight they provide into the design of both the mechanism and its control. Among successful examples are use of zero dynamics for the stabilization of walking and running behaviors (Westervelt et al. 2007; Chevallereau et al. 2009) as well as self-righting behaviors for the RHex hexapod (Saranli et al. 2004), both of which use sufficiently accurate dynamical models and subsequent high-bandwidth feedback to achieve stable and dynamic locomotory behaviors. Our contributions in the present paper not only provide a decompositional method that simplifies the design of such controllers, but also illustrate performance and gait-level controllability benefits associated with model-based feedback control.

There is also a large body of literature studying simpler, more fundamental models for basic locomotory behaviors, motivating our adoption of the Spring-Loaded Inverted Pendulum (SLIP) model. This model has received substantial attention in the literature, starting from its biological foundations (Blickhan and Full 1993), leading to its instantiation within dynamically dexterous monopods (Raibert 1986; Gregorio et al. 1997), followed by subsequent analysis (Schwind 1998; Altendorfer et al. 2004) and the design of associated gait controllers. Our treatment of the SLIP model also benefits from our recent work on its control through analytical return maps (Arslan et al. 2009; Ankarali et al. 2009). 
We continue the paper with a dimensionless model and control of the SLIP template in Sect. 2. We then present in Sect. 3, our embedding control framework in the context of a one-legged system that captures most relevant actuator limitations in the RHex platform except the pitch degree of freedom. We then proceed with the pronking controller for the full planar hexapod model in Sect. 4, followed by a characterization of its stability properties and sensitivity to different noise conditions in Sect. 5.

\section{The spring-loaded inverted pendulum template}

\subsection{Dimensionless system model and dynamics}

We model the SLIP dynamics as usual, consisting of a point mass $m$ and a freely rotating massless leg, endowed with a linear spring-damper pair of compliance $k_{s}$, rest length $l_{0}$ and, differently from similar models, viscous damping $d_{s}$. Throughout locomotion, the model alternates between stance and flight phases, which are further divided into the compression, decompression and ascent, descent subphases, respectively. Four important events define discrete transitions between these sub phases: touchdown, bottom, liftoff, and apex. During flight, the body is assumed to be a projectile acted upon by gravity, whereas in stance, the toe is assumed to be fixed on the ground and the mass feels radial forces generated by the leg. Table 1 details all relevant variables and parameters for the SLIP model which is illustrated in Fig. 1.

In order to eliminate redundant parameters and provide an efficient way to interpret our simulation results, we will use a dimensionless formulation of the dynamics both for the SLIP model and subsequent, more complex models. Redefining time as $t:=\bar{t} / \lambda$ with $\lambda:=\sqrt{l_{0} / g}$, scaling all distances with the spring rest length $l_{0}$ and using definitions detailed in Table 1, SLIP dynamics in dimensionless coordinates are given as

Flight: $\left[\begin{array}{c}\ddot{y} \\ \ddot{z}\end{array}\right]=\left[\begin{array}{c}0 \\ -1\end{array}\right]$,

$$
\begin{aligned}
\text { Stance: } & {\left[\begin{array}{l}
\ddot{\xi} \\
\ddot{\psi}
\end{array}\right] } \\
& =\left[\begin{array}{c}
\xi \dot{\psi}^{2}-\cos \psi-r_{s}(\xi-1)-c_{s} \dot{\xi} \\
(-2 \dot{\xi} \dot{\psi}+\sin \psi) / \xi
\end{array}\right] .
\end{aligned}
$$

Note that $(d / d t)^{n}=\lambda^{n}(d / d \bar{t})^{n}$ and all time derivatives in the above equations are with respect to the newly defined, scaled time variable. Throughout the rest of the paper, we will only work with dimensionless quantities and hence will not explicitly mention their dimensionless nature unless necessary.

\subsection{Deadbeat stride control for the SLIP template}

Gait-level control of SLIP hopping can be achieved with a variety of different control inputs (Schwind 1998; Zeglin 1999). As we describe in later sections, our embedding controller is based on the definition of a virtual SLIP, whose toe placement allows us to arbitrarily control its leg length at touchdown and liftoff instants. Consequently, in addition to the touchdown leg angle $\psi_{t}$, our gait controller for the template model uses leg lengths at touchdown and liftoff, $\xi_{t}$ and $\xi_{l}$ respectively, for stride control. This choice also makes stance dynamics fully passive, further simplifying controller design and improving embedding performance. A similar choice was made in our earlier work for the template based control of tripod running (Saranli and Koditschek 2003).

As usual, we summarize gait-level behavior of the SLIP model through a Poincaré section of its trajectories at each apex point with $\dot{z}=0$. Our stride controller hence seeks to regulate the discrete progression of the remaining states, the apex height $z_{a}$ and velocity $\dot{y}_{a}$. Specifically, we use a deadbeat gait controller (Saranli et al. 1998) based on an approximate but analytical stance map (Geyer et al. 2005; Arslan et al. 2009; Ankarali et al. 2009). In this context, given the current apex state $\left[\dot{y}_{a}, z_{a}\right]$, the deadbeat controller seeks to find control inputs $u:=\left[\psi, \xi_{t}, \xi_{l}\right]$ such that after a single step, a desired apex state $\left[\dot{y}_{a}^{*}, z_{a}^{*}\right]$ is achieved.
Table 1 State variables, parameters and the definitions of their dimensionless counterparts for the SLIP model. Variables with and without bars correspond to physical and dimensionless quantities, respectively

\begin{tabular}{llll}
\hline $\begin{array}{l}\text { Physical } \\
\text { quantity }\end{array}$ & $\begin{array}{l}\text { Dimensionless } \\
\text { group }\end{array}$ & Definition & Description \\
\hline $\bar{t}$ & $t$ & $:=\bar{t} / \lambda$ & Time (where $\left.\lambda:=\sqrt{l_{0} / g}\right)$ \\
{$[\bar{y}, \bar{z}]$} & {$[y, z]$} & $:=\left[\bar{y} / l_{0}, \bar{z} / l_{0}\right]$ & SLIP body position \\
{$[\bar{\xi}, \bar{\psi}]$} & {$[\xi, \psi]$} & $:=\left[\bar{\xi} / l_{0}, \bar{\psi}\right]$ & SLIP leg length and leg angle \\
$k_{s}$ & $r_{s}$ & $:=k_{s}\left(l_{0} /(m g)\right)$ & SLIP leg spring stiffness \\
$d_{s}$ & $c_{s}$ & $:=d_{s}\left(l_{0} /(\lambda m g)\right)$ & SLIP leg viscous damping \\
$\bar{F}$ & $F$ & $:=\bar{F} /(m g)$ & Force variables \\
$\bar{E}$ & $E$ & $:=\bar{E} /\left(m g l_{0}\right)$ & Energy variables \\
$\bar{p}_{\bar{\psi}}$ & $p_{\psi}$ & $:=\bar{p}_{\bar{\psi}}\left(\lambda /\left(m l_{0}^{2}\right)\right)$ & Angular momentum \\
\hline
\end{tabular}


Computation of leg lengths at touchdown and liftoff can easily be accomplished by using the energy difference between two successive apex states, computed as

$$
\Delta E:=\left(z_{a}^{*}-z_{a}\right)+\frac{1}{2}\left(\left(\dot{y}_{a}^{*}\right)^{2}-\left(\dot{y}_{a}\right)^{2}\right) .
$$

Depending on the sign of this desired energy change, we either inject energy into the system by precompressing the leg during flight $\left(\xi_{t}=1-\sqrt{2 \Delta E / r}\right.$ for $\left.\Delta E>0\right)$, or take out energy by prematurely lifting off with the spring still compressed $\left(\xi_{l}=1-\sqrt{2 \Delta E / r}\right.$ for $\left.\Delta E<0\right)$. Unfortunately, the computation of the remaining control input, the leg touchdown angle $\psi_{t}$, is not as straightforward and requires analytical approximations to the apex return map for the springmass hopper. This return map has three components: descent, stance and ascent, among which the first and last are trivially described by simple ballistic flight dynamics. However, the stance map is considerably more complicated, as evidenced by substantial work in the literature for its derivation in a simple enough, analytical form (Schwind 1998; Carver 2003; Geyer et al. 2005). In this paper, we use a modified version of the map described in Geyer et al. (2005), which we briefly review in the sequel.

Assuming that the leg stays close enough to the vertical, the effect of gravity during stance can be linearized, making both the angular momentum $p_{\psi}$ and the total mechanical energy constants of motion. Based on these assumptions and derivations similar to those described in Geyer et al. (2005), radial and angular stance trajectories in dimensionless coordinates take the form

$$
\begin{aligned}
\xi(t)= & 1+a+b \sin \left(\hat{\omega}_{0} t\right), \\
\psi(t)= & \psi_{t}+p_{\psi}(1-2 a)\left(t-t_{t}\right) \\
& +\frac{2 b p_{\psi}}{\hat{\omega}_{0}}\left[\cos \left(\hat{\omega}_{0} t\right)-\cos \left(\hat{\omega}_{0} t_{t}\right)\right],
\end{aligned}
$$

where $p_{\psi}:=\xi_{t}^{2} \dot{\psi}_{t}$ is the constant angular momentum and we define $\hat{\omega}_{0}:=\sqrt{r+3 p_{\psi}{ }^{2}}, \quad a:=\frac{p_{\psi}{ }^{2}-1}{\hat{\omega}_{0}^{2}}, \quad b:=$ $\sqrt{a^{2}+\left(2 E-p_{\psi}^{2}-2\right) / \hat{\omega}_{0}^{2}}$. Previously chosen leg lengths at touchdown and liftoff used as boundary conditions on (4) hence yield an approximate solution for the stance map. At this point, the descent, stance and ascent maps can be combined to provide an analytical return map $\left[\dot{y}_{a}, z_{a}\right]_{k+1}=$ $\hat{f}_{a}\left(\psi_{t},\left[\dot{y}_{a}, z_{a}\right]_{k}\right)$. Even though this map is not invertible in closed form, it is monotonic in $\psi_{t}$, admitting an easy numerical solution to the minimization problem

$$
\psi_{t}=\underset{\frac{-\pi}{2}<\psi<\frac{-\pi}{2}}{\operatorname{argmin}}\left(\dot{y}_{a}^{*}-\left(\pi_{\dot{y}_{a}} \circ \hat{f}_{a}\left(\psi_{t},\left[\dot{y}_{a}, z_{a}\right]_{k}\right)\right)\right)^{2},
$$

yielding an effective, step-based deadbeat controller for the SLIP model. We will use this controller to regulate the apex speed and height for the pronking behavior once proper embedding of SLIP dynamics within the hexapedal morphology is achieved.

\section{Dynamics and control of a torque actuated spring-mass Hopper}

Before we proceed with the planar pronking model, we introduce in this section an extended SLIP model with Torque actuation at the hip (SLIP-T) as a simpler intermediate model which captures most relevant actuator limitations of RHex, yet allows the main ideas for our embedding controller to be much more clearly explained. Section 4 will then extend these derivations to the planar hexapod morphology.

\subsection{System model and dynamics}

As illustrated in Fig. 2, the SLIP-T system is structurally similar to SLIP except that it incorporates a single motor at the hip with a controllable torque $\bar{\tau}$ instead of radial leg actuation. In order to make such a torque possible without adding an extra degree of freedom, we assume the presence of a rigid body with mass $m$, whose orientation is constrained to be horizontal (i.e. having infinite inertia). Finally, we also assume a very small mass $m_{t}$ attached to the toe to capture flight dynamics of the leg. In addition to possible physical instantiations of this model through explicit suppression of body pitch freedom (Sato and Buehler 2004), its main utility for us is the fact that it captures most of the attributes in RHex relevant to the dynamic embedding of SLIP, while being sufficiently simple to clarify the presentation of our method.

We define three different reference frames: a fixed inertial world frame $\mathcal{W}$, a body frame $\mathcal{B}$ attached to the body $\mathrm{COM}$ and finally a virtual toe frame, $\mathcal{V}$, marking the fixed location of the virtual SLIP toe on the ground during stance.
Fig. 2 SLIP-T: Spring-mass hopper with a fully passive leg and a rotary hip actuation
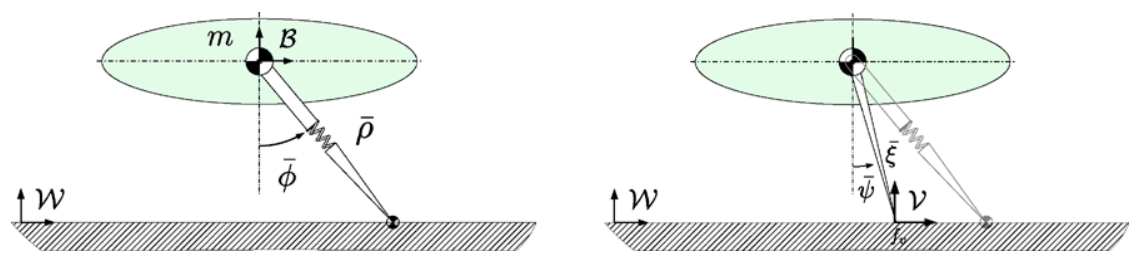
Table 2 State variables, parameters and the definitions of their dimensionless counterparts for the SLIP-T model. Variables with and without bars correspond to physical and dimensionless quantities, respectively

\begin{tabular}{lll}
\hline $\begin{array}{l}\text { Dimensionless } \\
\text { group }\end{array}$ & Definition & Description \\
\hline$\rho$ & $:=\bar{\rho} / l_{0}$ & Physical leg length \\
$\phi$ & $:=\bar{\phi}$ & Physical leg angle \\
$\mathbf{f}$ & $:=\overline{\mathbf{f}} / l_{0}$ & Physical toe position \\
$r$ & $:=k\left(l_{0} /(m g)\right)$ & Relative stiffness of the physical leg \\
$c$ & $:=d l_{0} /(\lambda m g)$ & Viscous friction of the physical leg \\
$\tau$ & $:=\bar{\tau} /\left(m g l_{0}\right)$ & Hip torque \\
$\eta_{t}$ & $:=m_{t} / m$ & Toe mass \\
\hline
\end{tabular}

$\mathcal{W}$ and $\mathcal{V}$ are coincident with the ground plane and all frames have identical orientations since the body angle is assumed constant. The toe location, length and hip angle for the physical leg are denoted with $\overline{\mathbf{f}}, \bar{\rho}$, and $\bar{\phi}$, respectively. The hybrid structure of the SLIP-T model is identical to the SLIP model described in Sect. 2.1, with an additional flag, $s$, defined to indicate whether the leg is in flight $(s=0)$ or in stance $(s=1)$.

In our derivation of the dimensionless equations of motion for the SLIP-T model, the definitions of Table 1 will be used for the virtual leg defined between the body and virtual toe frames. Moreover, we will also use additional definitions listed in Table 2 for the physical SLIP-T leg.

Within a Newton-Euler framework, the radial springdamper force $F_{r}:=-r(\rho-1)-c \dot{\rho}$, the effect of the hip torque $F_{\tau}:=-\tau / \rho$, acting orthogonally to $F_{r}$ and the gravitational acceleration constitute the only external forces acting on the body during stance. The total force vector exerted on the body by the leg during stance can be formulated as

$\mathbf{F}=R(\phi)\left[\begin{array}{c}F_{\tau} \\ F_{r}\end{array}\right]$,

where $R(\phi)$ denotes the rotation matrix that determines the orientation of the leg with respect to $\mathcal{B}$. Combining (7) with flight dynamics and by making use of the touchdown flag $s$, we can obtain the overall SLIP-T equations of motion as

$$
\begin{aligned}
& {\left[\begin{array}{l}
\ddot{y} \\
\ddot{z}
\end{array}\right]=s R(\phi)\left[\begin{array}{c}
-\tau / \rho \\
-r(\rho-1)-c \dot{\rho}
\end{array}\right]+\left[\begin{array}{c}
0 \\
-1
\end{array}\right],} \\
& \eta_{t} \ddot{\mathbf{f}}=(s-1) R(\phi)\left[\begin{array}{c}
-\tau / \rho \\
-r(\rho-1)-c \dot{\rho}
\end{array}\right] .
\end{aligned}
$$

\subsection{Virtual foot placement and virtual toe coordinates}

Clearly, control inputs available to SLIP-T are not fully compatible with those that we used to perform gait control on the SLIP template. Even though the touchdown angle can be realized within the SLIP-T model by controlling leg angle during flight, it is unclear how the touchdown and liftoff leg lengths can be commanded in the absence of any radial leg actuation. Moreover, any attempt to use the hip torque will substantially change the angular momentum around the toe of the SLIP-T, pushing its dynamics farther from the SLIP template.

Fortunately, both of these problems can be addressed with the realization that the desired SLIP template does not need to exactly coincide with the physical leg of the SLIP-T model. As evident from the illustration in Fig. 2, when the virtual toe position $\mathbf{f}_{v}$ is different than the physical toe position $\mathbf{f}$, the virtual leg length also ends up being different than the physical leg length. Consequently, if we use the hip motor during flight ${ }^{1}$ to bring the physical leg angle to $\phi_{t}=\arccos \left(\xi_{t} \cos \left(\psi_{t}\right)\right)$, we can achieve both $\xi_{t}$ and $\psi_{t}$ by choosing the virtual toe position as $\overline{\mathbf{f}}_{v}=\left[y+\xi_{t} \cos \left(\psi_{t}\right), 0\right]$, determining the position of the frame $\mathcal{V}$ for the following step. Note, however, that the state of the physical leg at touchdown is determined by the flight dynamics and may not exactly match the commanded angle due to the small but finite toe mass. In such cases, our choice of the virtual toe position prioritizes the desired SLIP touchdown angle over its leg length and uses adjusted versions of the touchdown SLIP states with $\tilde{\psi}_{t}=\psi_{t}$ and $\tilde{\xi}_{t}=z_{t} / \cos \psi_{t}$.

Following the placement of the virtual toe frame $\mathcal{V}$, we define a new set of dimensionless polar coordinates for the stance dynamics in which the SLIP embedding will take place, defined as

$\mathbf{c}_{v}:=[\xi, \psi]^{T}$.

3.3 Control of stance dynamics trough active embedding of the ideal SLIP

The stance dynamics of SLIP-T in virtual toe coordinates are given by

$\ddot{\xi}=\xi \dot{\psi}^{2}-\cos \psi+K_{\xi}$,

$\ddot{\psi}=\frac{-2 \dot{\xi} \dot{\psi}+\sin \psi}{\xi}+K_{\psi} /\left(\xi^{2}\right)$,

where $K_{\xi}$ and $K_{\psi}$ capture the effect of both the physical leg spring and the external hip torque on the virtual toe coordinates and can be written as

$\mathbf{K}:=\left[K_{\xi}, K_{\psi}\right]^{T}=\left(D_{c} \phi\right) \tau+\left(D_{c} \rho\right) F_{r}$,

where $D_{c} \phi:=[\partial \phi / \partial \xi, \partial \phi / \partial \psi]^{T}$ and $D_{c} \rho:=[\partial \rho / \partial \xi$, $\partial \rho / \partial \psi]^{T}$ denote Jacobian matrices of the hip angle and leg

\footnotetext{
${ }^{1} \mathrm{~A}$ simple PD law can be used to this end for controlling the hip motor during flight.
} 
length with respect to virtual leg coordinates. For simplicity, we define $\mathbf{J}:=\left(D_{c} \phi\right)$ and $\mathbf{B}:=\left(D_{c} \rho\right) \mathbf{F}_{r}$. The primary goal of our embedding controller is to find appropriate hip controls to force the dynamics of (11) and (12) to match the simple SLIP dynamics in (2). Simple inspection reveals that this can only be accomplished if we have

$\mathbf{K}=[U(\xi), 0]$,

where $U(\xi)$ is the desired radial potential law for the SLIP template and the second component enforces conservation of angular momentum around the virtual toe.

Unfortunately, the SLIP-T model has only a single actuator, meaning that both components of $\mathbf{K}$ cannot be independently controlled. Moreover, particularly when the virtual toe is close to the physical toe, radial control affordance on $\mathbf{K}$ is very low. Consequently, we choose to focus explicit control effort on the angular dynamics and attempt to preserve angular momentum around the virtual toe with

$\tau=J_{\psi}^{-1}\left(0-B_{\psi}\right)=-\rho \tan (\psi-\phi) F_{r}$,

where $J_{\psi}$ and $B_{\psi}$ denote rows of $\mathbf{J}$ and $\mathbf{B}$ associated with the $\psi$ coordinate, respectively. Our assumption is that if the physical leg compliance (i.e. the passive dynamics of the robot) are properly chosen, they will approximately yield the desired result for the remaining coordinate in the virtual leg coordinates.

\subsection{Gait level control of SLIP-T: energy corrections}

Not surprisingly, our choice of prioritizing angular dynamics over radial dynamics in (15) causes the SLIP embedding to perform poorly in regulating the total energy in the system, which depends mostly on the radial spring dynamics. This necessitates modifications in our embedding algorithm to account for energetic errors introduced by both radial inaccuracies as well as the presence of damping.

Our corrections primarily target the desired energy change of (3). For the SLIP-T model, we need to also supply the energy lost through damping, $\Delta E_{\text {loss }}$, with

$\Delta E=\left(z_{a}^{*}-z_{a}\right)+\frac{1}{2}\left(\left(\dot{y}_{a}^{*}\right)^{2}-\left(\dot{y}_{a}\right)^{2}\right)+\Delta E_{\text {loss }}$.

Unfortunately, accurate estimation of damping losses is a hard problem and depends critically on physical implementation details. Even under simple viscous damping, it is not possible to obtain a sufficiently accurate analytic solution. Fortunately, radial stance trajectories of both the SLIP-T model, as well as the pronking behavior of later sections do not exhibit significant variability across strides in their damping losses. Consequently, we use a sinusoidal fit, inspired by the form of (4), to measured radial trajectories within each step to estimate the damping losses within the next stance phase. As shown in Sect. 5, this yields excellent results at steady state, as well as very good performance even during transients.

A more important source of inaccuracy in the overall performance of the embedding controller is how the touchdown and liftoff leg lengths are chosen to realize the desired energy change. Since the radial dynamics of the embedding deviate from the fully passive stance dynamics of the ideal SLIP model, the computations of Sect. 2.2 are not good enough and a better analysis is needed for the energy supplied by the hip torque:

$$
\begin{aligned}
\Delta E & =\int_{t_{t}}^{t_{l}} \tau \dot{\phi}(t) d t \\
& =\int_{t_{t}}^{t_{l}}-\rho(t) \tan (\psi(t)-\phi(t)) F_{r}(t) \dot{\phi}(t) d t .
\end{aligned}
$$

Having already compensated for damping, we can assume that $F_{r}(t)=-r(\rho(t)-1)$ to yield

$\Delta E=\int_{t_{t}}^{t_{l}} \rho(t) \tan (\psi(t)-\phi(t)) \dot{\phi}(t) r(\rho(t)-1) d t$,

which, despite the availability of analytical approximations to all of its components through (4) and (5), still does not admit an exact analytic solution. Nevertheless, we propose an approximation to this integral to further improve on the poor energetic performance arising from deploying the ideal SLIP energy control. We first assume that $(1-\rho) \approx(1-\xi)$, which is reasonable if the desired changes in gait parameters are not too dramatic. Moreover, the angle difference between the physical and virtual leg stays relatively constant throughout stance and can be approximated on the average with its value at bottom. This yields an approximation to the integral in (18) as

$$
\Delta E \approx \int_{t_{t}}^{t_{l}} r(\xi(t)-1) \tan \left(\psi_{b}-\phi_{b}\right) \rho_{b} \dot{\phi}_{b} d t,
$$

which, once the radial solution of (4) is plugged in, reduces to

$$
\begin{aligned}
\Delta E \approx & r \tan \left(\psi_{b}-\phi_{b}\right) \rho_{b} \dot{\phi}_{b} \\
& \times\left(a\left(t_{t}-t_{l}\right)-b\left(\cos \left(\hat{\omega}_{0} t_{t}\right)-\cos \left(\hat{\omega}_{0} t_{l}\right)\right) / \hat{\omega}_{0}\right),
\end{aligned}
$$

where $a, b, \hat{\omega}_{0}$ and event times are all as defined in Sect. 2.2 and are functions of the control inputs. In order to avoid numerically solving this equation in multiple dimensions, we recall our observation that the angular dynamics do not substantially effect the radial, energetic behavior of the system. Consequently, we modify (20) to use the neutral touchdown angle $\psi_{n}:=\left\{\psi_{t} \mid\left[\dot{y}_{a}, z_{a}\right]^{T}=\hat{f}_{a}\left(\psi_{t},\left[\dot{y}_{a}, z_{a}\right]^{T}\right)\right\}$ as one of the input commands, yielding a one dimensional analytic equation, which we then solve for $\xi_{t}$ to achieve the desired 
pumping energy. Once the appropriate leg length is determined, the deadbeat controller of Sect. 2.2 is used to find the corresponding touchdown angle.

\section{Dynamics and control of planar hexapedal pronking}

As described earlier, our target experimental platform for the pronking behavior in the long run is RHex, an autonomous hexapod robot with only a single rotary actuator on each hip. When contralateral legs on this platform are used in synchrony for behaviors such as the pronk, the sprawled posture of the morphology ensures that locomotion dynamics live on the saggital plane. Consequently, a saggital planar model is often capable of capturing relevant aspects of the dynamics for the purposes of modeling and analyzing such behaviors (Saranli et al. 2004; Greenfield et al. 2005). In this section, we describe and use such a planar model, Slimpod (Saranli 2000, 2002), to design a feedback controller for pronking.

\subsection{System model and dynamics}

The Slimpod model, illustrated in Fig. 3, consists of a rigid body with inertia $I$ and mass $m$, to which three compliant legs, each representing a saggitally symmetric pair of legs on RHex, are attached. The position and orientation of the body are represented by a body-fixed frame $\mathcal{B}$ with respect to an inertial world frame $\mathcal{W}$. As in Sect. 3.1, we also define a "virtual leg" extending from the body center of mass (COM) to a stationary point on the ground coincident with the virtual toe frame $\mathcal{V}$ having the same orientation as the world frame. Legs are considered massless during stance, with the toe position fixed on the ground at $\overline{\mathbf{f}}_{i}$, but very small toe masses $m_{t} \ll m$ are used to represent protraction dynamics during flight. Each leg is attached to the body through a pin joint with independently controllable torque $\bar{\tau}_{i}$, located at $\overline{\mathbf{a}}_{i}$ in body coordinates. Each leg is compliant with stiffness $k_{i}$ and incorporates viscous damping with coefficient $d_{i}$.

As in previous sections, we will work in dimensionless coordinates for the Slimpod model. To this end, in addition to variables defined in Tables 1 and 2, we will also use Slimpod-specific definitions detailed in Table 3. The derivation of the hybrid dynamics for the planar hexapod model closely parallels the presentation in Saranli (2002), which we omit in the present paper for space considerations.

\subsection{Controlling Slimpod stance dynamics trough an embedding of the ideal SLIP}

In controlling the stance dynamics of the Slimpod model for the pronking behavior, we use an embedding controller very similar to the controller presented in Sect. 3.3. However, the presence of three individual legs as well as the pitch degree of freedom necessitates a number of important extensions.

Firstly, we consider the SLIP template to have transitioned into stance as soon as at least one of the Slimpod legs touches the ground. This event also triggers the placement of the virtual toe and defines new virtual toe coordinates in the frame $\mathcal{V}$, now extended with the pitch degree of freedom to yield

$\mathbf{c}_{v}=[\xi, \psi, \alpha]^{T}$.

Normally, the flight controller is responsible for servoing individual Slimpod legs to proper locations to achieve the desired touchdown state for the SLIP template. However, as a result of the nontrivial flight dynamics of Slimpod legs and the body, actual touchdown states of the template may not be exact. In such cases, as in Sect. 3.2,
Fig. 3 Slimpod: A planar dynamic model for hexapedal pronking

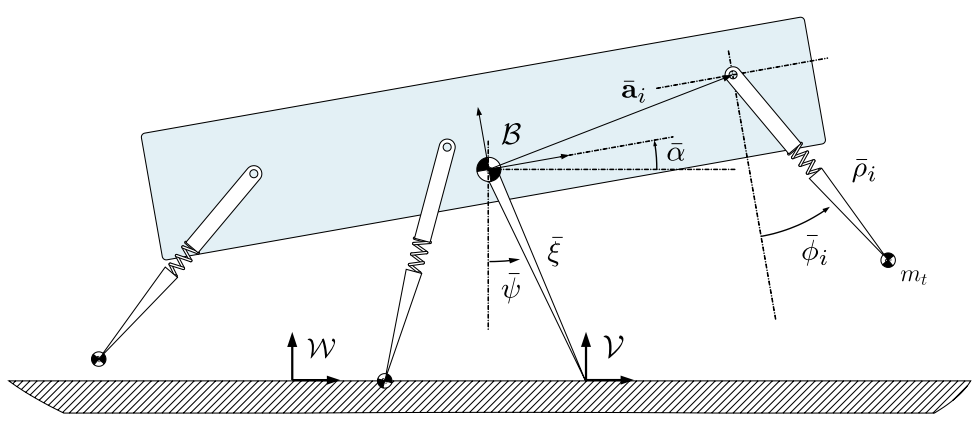

Table 3 Dimensionless parameters and variables of slimpod model

\begin{tabular}{lll}
\hline Dimensionless var. or par. & Derivation & Definition \\
\hline$\alpha$ & $\bar{\alpha}$ & Dimensionless body orientation \\
$j$ & $I /\left(m l_{0}^{2}\right)$ & Dimensionless inertia \\
$\mathbf{a}_{i}$ & $\overline{\mathbf{a}}_{i} / l_{0}$ & Dimensionless hip position (in $\mathcal{B})$ \\
\hline
\end{tabular}


we prioritize the touchdown angle over the touchdown length.

Following the placement of the virtual toe, the stance controller takes over and attempts to mimic ideal SLIP template dynamics by properly choosing hip torque inputs of the Slimpod model. As in Sect. 3.3, we start by writing down the stance equations of motion in virtual toe coordinates to yield

$\ddot{\xi}=\xi \dot{\psi}^{2}-\cos \psi+K_{\xi}$,

$\ddot{\psi}=\frac{-2 \dot{\xi} \dot{\psi}+\sin \psi}{\xi}+K_{\psi}$,

$\ddot{\alpha}=\frac{K_{\alpha}}{j}$,

which are identical with the SLIP-T dynamics of (11) and (12) with the addition of pitch dynamics. The forcing vector $\mathbf{K}:=\left[K_{\xi}, K_{\psi}, K_{\alpha}\right]^{T}=\left(D_{c} \phi\right) \boldsymbol{\tau}_{s}+\left(D_{c} \rho\right) \mathbf{F}_{r, s}$ captures the effect of both the hip torques $\boldsymbol{\tau}_{s}$ and radial leg forces $\mathbf{F}_{r, s}$ on each virtual toe coordinate with $D_{c} \phi$ and $D_{c} \rho$ denoting Jacobian matrices of the leg angles and lengths with respect to virtual toe coordinates. As in Sect. 3.3, we define $\mathbf{J}:=$ $D_{c} \boldsymbol{\phi}$ and $\mathbf{B}:=\left(D_{c} \boldsymbol{\rho}\right) \mathbf{F}_{r, s}$.

We seek to find appropriate hip torques to force the Slimpod center of mass to obey the dynamics of the SLIP template with

$\mathbf{K}^{*}=\left[U^{*}(\xi), 0, M_{\alpha}^{*}\right]$

where $U^{*}(\xi)$ is the desired radial spring potential law, the second component forces angular SLIP dynamics and $M_{\alpha}^{*}=$ $-K_{\alpha} \alpha-K_{\dot{\alpha}} \dot{\alpha}$ is chosen as a simple PD law for pitch stabilization.

Unfortunately, as described in Saranli and Koditschek (2003), J is rank deficient for configurations in which all legs are parallel, making this simple inversion impossible. The rank deficiency becomes even worse when the legs are vertical, reducing control affordance to a single degree of freedom. Since the pronking behavior inevitably must go through such configurations, we will address this problem in the next section by prioritizing appropriate coordinates of the SLIP template while also respecting motor torque limits.

\subsubsection{Handling singularities, torque limits and partial stance}

The rank deficiency of $\mathbf{J}$ for the planar hexapod model is very similar to the lack of radial control affordance in Sect. 3.3, where our solution was to rely on the passive dynamics of the morphology to realize the desired radial dynamics. Since all legs in the Slimpod model incorporate passive compliance, this will still be possible, allowing us to focus active control effort on angular SLIP dynamics for the embedding. We will initially assume that all three legs are in contact with the ground and introduce exceptions later to deal with partial touchdown and liftoff. As such, when the radial component is excluded from the inversion, the inverse dynamics controller attempts to simultaneously satisfy both angular template dynamics and pitch stabilization with

$$
\begin{aligned}
\boldsymbol{\tau}_{\psi, \alpha}(v):= & \mathbf{J}_{\psi, \alpha}^{T}\left(\mathbf{J}_{\psi, \alpha} \mathbf{J}_{\psi, \alpha}^{T}\right)^{-1} \\
& \times\left(\left[0 M_{\alpha}^{*}\right]^{T}-\mathbf{B}_{\psi, \alpha}\right)+\mathbf{J}_{\psi, \alpha}^{\perp} v,
\end{aligned}
$$

where $\mathbf{J}_{\psi, \alpha}^{\perp}$ spans the nullspace of $\mathbf{J}_{\psi, \alpha}$ and $v$ covers the remaining freedom.

In order to ensure practical applicability of our controller, we also impose an additional constraint on hip torques based on RHex's actuator torque-speed characteristics and a consideration of premature leg liftoff which may cause undesirable loss of actuator affordance. Formally, we specify these constraints individually for each leg, yielding the allowable torque space

$\mathcal{T}_{\text {lim }}:=\left\{\boldsymbol{\tau} \mid \tau_{i, \min } \leq \tau_{i} \leq \tau_{i, \max }, 1 \leq i \leq 3\right\}$

whose intersection with the solution space of (26) is used by our controller to yield

$\boldsymbol{\tau}_{s}=\underset{\boldsymbol{\tau}_{\psi, \alpha}(v) \in \mathcal{T}_{\text {lim }}}{\arg \min }\left\|\boldsymbol{\tau}_{\psi, \alpha}(v)\right\|$,

which can easily be solved using linear programming. Unfortunately, there are situations where the desired solution space and the feasible torque space do not intersect. In such cases, we prioritize the preservation of angular momentum around the virtual toe, using the alternative torque solution

$\boldsymbol{\tau}_{\psi}(w):=\mathbf{J}_{\psi}^{T}\left(\mathbf{J}_{\psi} \mathbf{J}_{\psi}^{T}\right)^{-1}\left(\left[0 M_{\alpha}^{*}\right]^{T}-\mathbf{B}_{\psi}\right)+\mathbf{J}_{\psi}^{\perp} w$,

where $\mathbf{J}_{\psi}^{\perp}$ spans the nullspace of $\mathbf{J}_{\psi}$ and $w$ covers the remaining freedom. This yields a new form for the overall solution as

$\boldsymbol{\tau}_{s}=\underset{\boldsymbol{\tau}_{\psi}(w) \in \mathcal{T}_{\text {lim }}}{\arg \min }\left\|\boldsymbol{\tau}_{\psi}(w)\right\|$,

which is, once again, easily solvable using linear programming (Saranli 2002).

The controller that results from using the solutions of (28) and (30) is applicable when at least two legs are in stance. However, close to the touchdown and liftoff events, and particularly in the presence of noise, the robot may find itself with only a single leg in contact with the ground. Earlier work on pronking (McMordie and Buehler 2001) and our simulations show that pitch instability induced by such underactuated phases is a significant mode of failure. Moreover, when a single leg is in stance, control affordance is primarily in the pitch degree of freedom. Consequently, for 
single-leg configurations, we only attempt to stabilize the pitch with

$\tau_{s}=J_{\alpha}^{-1}\left(M_{\alpha}^{*}-B_{\alpha}\right)$

which yields a scalar torque for the single leg and prevents problematic loss of pitch stability.

\subsection{Gait level control of the Slimpod}

As a result of the pitch stabilizing force imposed by $M_{\alpha}^{*}$, pitch oscillations during steady-state pronking are expected to be very small. Consequently, the stance dynamics for the Slimpod are expected to closely mirror those of the SLIP-T model. As a result, we will use the gait controller developed in Sect. 3.4 with only a few minor extensions for step-wise control of pronking.

Firstly, we choose the compliance and damping parameters of the gait controller to reflect the presence of three legs acting in parallel:

$d=\sum_{i=1}^{3} d_{i}, \quad k=\sum_{i=1}^{3} k_{i}$.

We also coordinate flight control of all legs to ensure simultaneous touchdown of all three legs, making sure that desired SLIP control inputs provided by the gait level controller in Sect. 4.3 can be realized by explicit placement of the virtual toe. To this end, the flight controller continuously solves kinematic equations for all legs and servoes them to their required positions with respect to the world frame as illustrated in Fig. 4. Based on the SLIP control decisions $\psi_{t}$ and $\xi_{t}$, target leg angles are given by

$\overline{\mathbf{p}}_{i}=\xi_{t}\left[\begin{array}{c}\sin \psi_{t} \\ \cos \psi_{t}\end{array}\right]+R\left(\alpha_{t}\right) \mathbf{a}_{i}$,

$\phi_{i t}^{*}=\arccos \left(p_{z i}\right)-\alpha_{t}$,

where $\overline{\mathbf{p}}_{i}$ are the positions of the hips in $\mathcal{V}$ for each leg and $\phi_{i t}^{*}$ are the target leg angles.

All of our pronking simulations presented in Sect. 5 use this flight controller, together with the embedding stance controller described in Sect. 4.2.

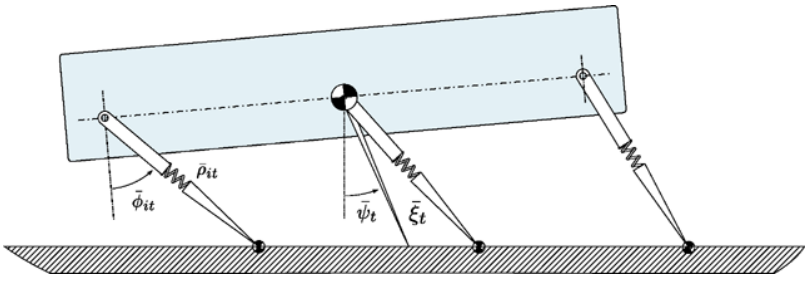

Fig. 4 Leg kinematics at the time of touchdown

\section{Simulation studies}

In this section, we provide simulation evidence to illustrate that the embedding controllers described in Sects. 3 and 4 for the SLIP-T and Slimpod models, respectively, are capable of producing stable and controllable pronking. For hexapedal pronking, we also characterize the robustness of the resulting behavior against modeling errors in the form of parameter mismatch, sensor noise in the form of state measurements polluted by white Gaussian noise and actuation noise in the form of piecewise constant torque outputs updated at $1 \mathrm{KHz}$. To this end, we measure steady-state tracking performance as a function of noise magnitude and show that an experimental implementation of the proposed pronking controller is feasible under realistic sensory performance.

All simulations were run in Matlab, using a hybrid dynamical simulation toolkit based on SimSect (Saranli 2000), whose qualitative correspondence to the physical performance of RHex was previously verified (Saranli 2002; Saranli et al. 2004). All kinematic and dynamic parameters for both the SLIP-T and Slimpod models, detailed in Table 4, were chosen to closely match the physical RHex robot to ensure future applicability of our results to an experimental implementation. ${ }^{2}$

\subsection{Existence and nature of stable limit cycles}

We first investigate whether our embedding controller leads to a stable limit cycle within the state space of the system. Figures 5 and 6 illustrate example runs for the SLIP-T and Slimpod models, respectively, starting from same initial condition and converging to the same selected goal state of $z=1.15, \dot{y}=1.1$ (corresponding to a physical goal of $\bar{z}=20.125 \mathrm{~cm}$ and $\dot{\bar{y}}=1.44 \mathrm{~m} / \mathrm{s}$ for the RHex platform, with a leg length of $l_{0}=17.5 \mathrm{~cm}$-Saranli et al. 2001). In both figures, left two plots show forward velocity and body height as a function of dimensionless time, while the rightmost plots show the progression of apex states at each step. These figures clearly show that models converge to a limit cycle with very small steady-state errors indicating that the combination of the embedding controller with the SLIP deadbeat controller successfully stabilizes locomotion.

In all of our simulations, we observed that the models either converge to a single, stable, period-one limit cycle, or irrecoverably fail due to transitional faults such as toe stubbing or pitch oscillations leading to the body colliding with the ground. Convergence speed is primarily determined by manually tuned limits we impose on the touchdown leg length $\left(\xi_{t}>0.9\right)$, which also limits the energy that can be

\footnotetext{
${ }^{2}$ Note that the results are applicable to a wide range of parameter combinations due to our dimensionless formulation of the models.
} 
Table 4 Kinematic and dynamic parameters for the Slimpod and SLIP-T models. All quantities are in dimensionless units. The single SLIP-T leg represents all six of RHex's legs, while each Slimpod leg represents a pair of contralateral legs on RHex

\begin{tabular}{lllllllll}
\hline & $r_{i}$ & $c_{i}$ & $\bar{\tau}_{\max }$ & $\dot{\phi}_{\max }$ & $\mathbf{a}_{1}$ & $\mathbf{a}_{2}$ & $\mathbf{a}_{3}$ & $j$ \\
\hline SLIP-T & 1.11 & 25.9 & 5.91 & 6.88 & - & - & - \\
Slimpod & 0.186 & 8.62 & 2.19 & 6.88 & {$[-1.26,0]$} & {$[0,0]$} & {$[0,1.26]$} & 0.62 \\
\hline
\end{tabular}

Fig. 5 An example SLIP-T simulation with $t_{\text {end }}=30$ $\left(\bar{t}_{\text {end }}=4 \mathrm{~s}\right.$ for RHex), starting from an initial condition of $z=1.4, \dot{y}=0.9$, towards an apex goal $z^{*}=1.15, \dot{y}^{*}=1.1$

Fig. 6 An example pronking simulation with $t_{\text {end }}=30$ $\left(\bar{t}_{\text {end }}=4 \mathrm{~s}\right.$ for RHex $)$, starting from an initial condition of $z=1.4, \dot{y}=0.9, \dot{\alpha}=0$, towards an apex goal $z^{*}=1.15$, $\dot{y}^{*}=1.1$
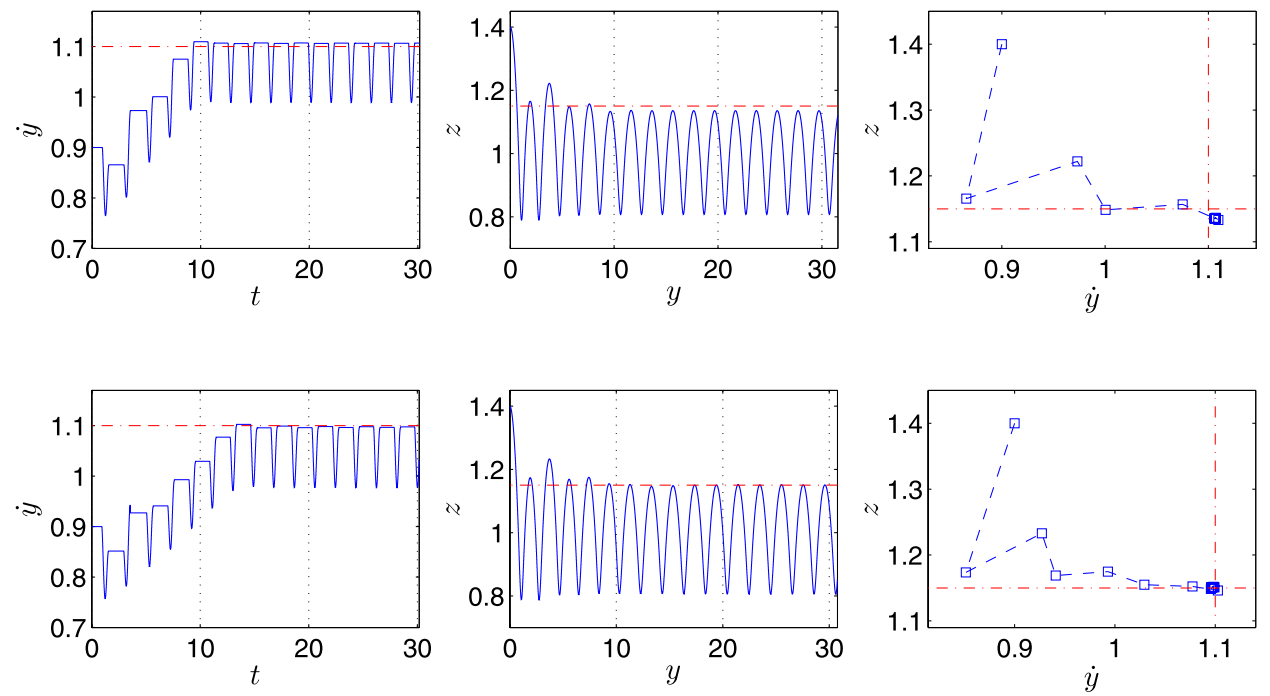

injected into the system at every step. Smaller touchdown lengths for the virtual leg were found to cause pitch instability due to excessive energy input leading to decreased embedding performance. In order to prevent steps inputs with large magnitude, we also use a reference governor on the forward velocity command that also effects convergence speed. Finally, it is worth noting that the state progressions for both the SLIP-T and Slimpod models are very similar, suggesting that the desired SLIP template was indeed correctly embedded.

\subsection{Stability and basins of attraction}

In order to generalize our observations in Sect. 5.1 and more accurately characterize stability properties of both the SLIP-T and Slimpod controllers, we systematically ran simulations from a variety of different initial conditions toward a single common goal of $z^{*}=1.16, \dot{y}^{*}=1.1$ (corresponding to $\bar{z}_{a}^{*}=0.203 \mathrm{~cm}$ and $\dot{\bar{y}}_{a}^{*}=1.44 \mathrm{~m} / \mathrm{s}$ for RHex). Each individual run with $t_{\text {end }}=52\left(\bar{t}_{\text {end }}=7 \mathrm{~s}\right.$ for RHex $)$ was considered stable if the last five apex states were within $1 \%$ of their average.

Figure 7 shows the resulting domain of attraction for SLIP-T running under the action of our controller. Stable locomotion cannot be achieved at very high speeds, which is expected due to the torque limits we impose on the actuators. Similarly, low speeds are problematic since the effects of hip torques are primarily in the horizontal direction

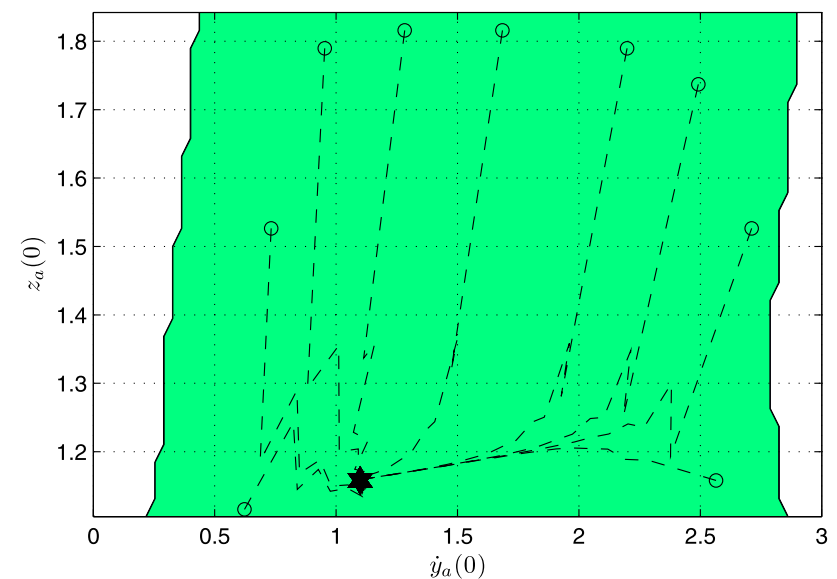

Fig. 7 (Color online) Stable domain of attraction for the SLIP-T model towards the goal $\dot{y}_{a}^{*}=1.1$ and $z_{a}^{*}=1.16$. The shaded (green) region shows initial conditions from which locomotion converges to a stable limit cycle. Dashed lines illustrate a few example runs to show convergence behavior

for narrow leg angles associated with slow speeds, making it impossible to inject vertical energy into the system. Our controller successfully stabilizes running for the large range of speeds in between, also covering a large range of initial heights.

Similarly, Figs. 8 and 9 illustrate two cross sections of the domain of attraction for the Slimpod model, whose state space now has the additional pitch degree of freedom. 


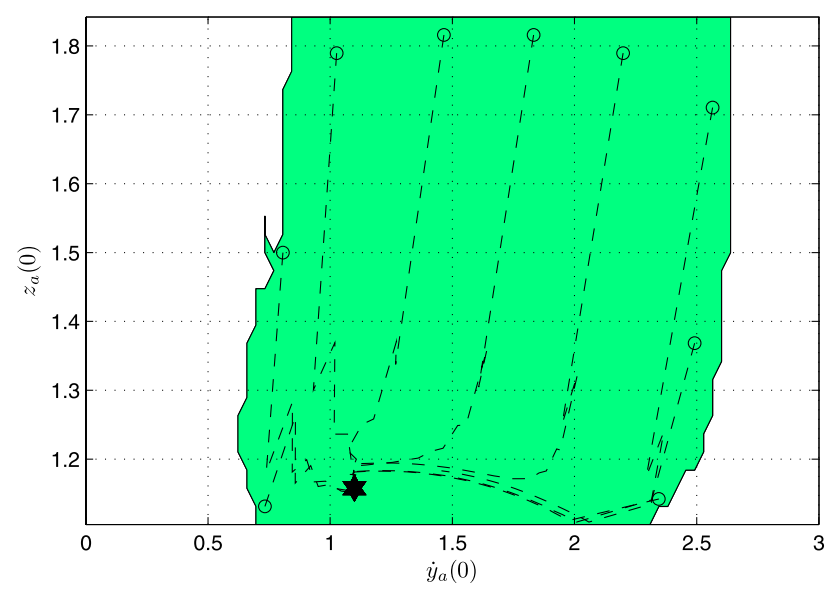

Fig. 8 (Color online) Cross section $\left(\dot{y}_{a}-z_{a}\right)$ of the domain of attraction towards the goal $\dot{y}_{a}^{*}=1.1$ and $z_{a}^{*}=1.16$. The shaded (green) region illustrates initial conditions from which the hexapod converges to stable pronking. Dashed lines illustrate a few example runs to show convergence behavior

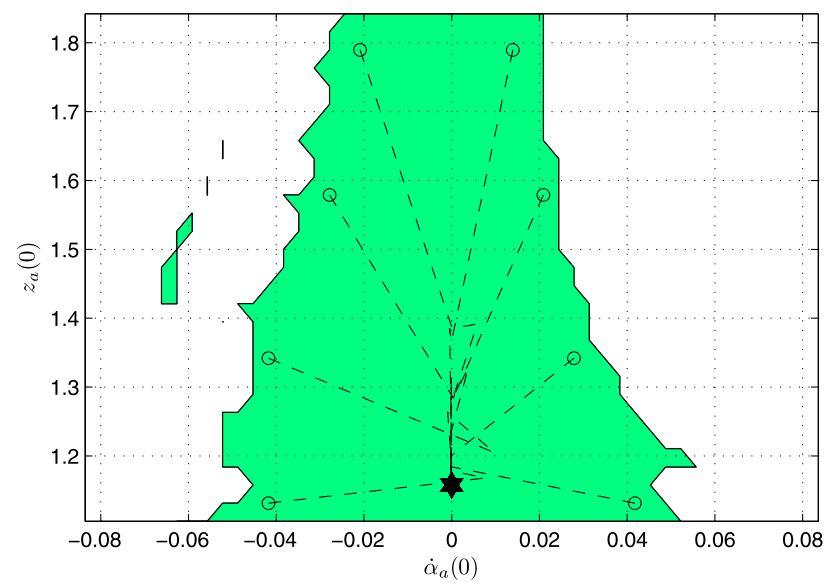

Fig. 9 (Color online) Cross section $\left(\dot{\alpha}_{a}-z_{a}\right)$ of the stable domain of attraction towards the goal $\dot{y}_{a}^{*}=1.1$ and $z_{a}^{*}=1.16$. The shaded (green) region illustrates initial conditions from which the hexapod converges to stable pronking. Dashed lines illustrate a few example runs to show convergence behavior

Not surprisingly, it is slightly harder to stabilize hexapedal pronking due to the additional pitch degree of freedom, leading to a smaller domain of attraction. Nevertheless, the stable domain for the pronking controller is still large enough to admit practical deployment.

\subsection{Gait-level controllability}

As we noted before, an important novelty of template based control is its provision of a simple, task specific interface for high level control of locomotion. In contrast to existing pronking controllers in the literature, this approach provides a high degree of control authority for the pronking gait with independently adjustable forward speed and hopping height.

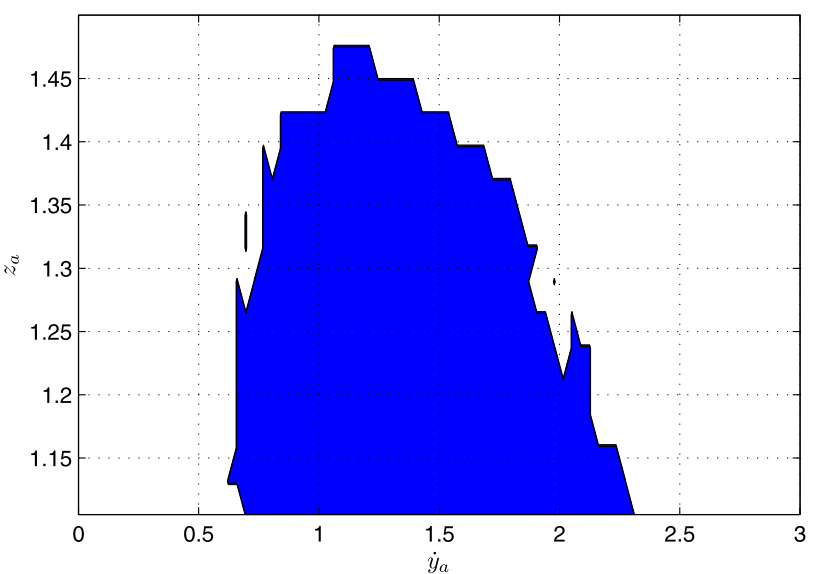

Fig. 10 (Color online) Gait-level controllability of the pronking controller. The shaded (blue) region illustrates the set of apex goal settings for which stable pronking is possible and steady-state was within $5 \%$ of the desired goal. All points in this goal region use the same kinematic and dynamic parameters given in Table 4

In order to characterize the extent to which high-level gait parameters can be controlled for the pronking gait, we ran a series of simulations with different apex goal settings from a rectangular region in the apex state space. Each run was started from an initial condition close to the goal and the stability criteria of the previous section were used to determine successful runs. Moreover, we also checked whether the hexapod was able to reach steady-state at least within 5\% of the desired goal state. Under these criteria, Fig. 10 shows all goal states that are successfully stabilized by the embedding controller for pronking with the Slimpod model.

These results show that the embedding controller is not only capable of stabilizing isolated goal settings, but that there is a large, contiguous range of goal states that can explicitly be requested by a high-level controller. Such gaitlevel control authority is essential if dynamic behaviors such as pronking are to be deployed in complex terrain which would require rapid and stable adjustment of gait parameters to successfully overcome obstacles and choose proper footholds.

\subsection{Sensitivity analysis}

Any physical implementation of our embedding controller will inevitably have to deal with several sources of noise and uncertainty. First and foremost, inaccuracies in measuring the kinematic and dynamic parameters of the platform may have considerable impact on controller performance. Moreover, digital torque control is often limited to piecewise constant output as opposed to the continuous torque profile required by (26). Finally, state feedback in a robotic platform requires the processing of sensory information, involving varying levels of noise both due to imperfect sensors as well as the approximate nature of estimation filters. In this 
Fig. 11 Sensitivity of steady-state tracking performance of the pronking controller with respect to a miscalibrated relative spring stiffness $\hat{r}_{i}$
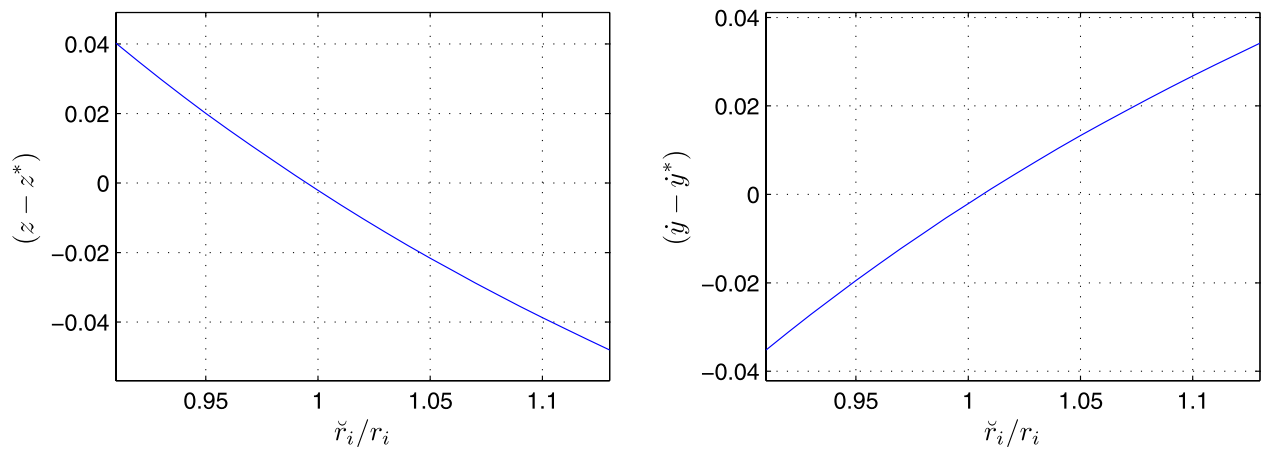

section, we characterize the sensitivity of our pronking controller against all these three sources of uncertainty.

\subsubsection{Sensitivity to model uncertainty}

Among most important and difficult to measure parameters for the Slimpod model are the coordinates of the leg attachment points $\mathbf{a}_{i}$ with respect to the center of mass, and the relative leg spring stiffness $r_{i}$. Moreover, initial estimates of these parameters may become more inaccurate as a result of material fatigue and structural changes in the robot after continuous use on complex terrain. Consequently, we first investigate the impact of an increasing discrepancy between the real and assumed values of these parameters on the tracking accuracy of our pronking controller.

Figure 11 illustrates the impact of inaccurate leg stiffness values on the steady-state tracking performance of the pronking controller, where $\hat{r}_{i}$ denotes the stiffness value assumed by the controller whereas $r_{i}$ is the actual spring stiffness. The tracking performance was characterized by comparing apex height and speed parameters associated with stead-state limit cycle, $z$ and $\dot{y}$, with their commanded values, $z^{*}$ and $\dot{y}^{*}$. These results show that pronking remains stable even in the presence of up to $10 \%$ error in the spring stiffness. Note that the approximate nature of our controller causes some steady state bias even when $\hat{r}_{i} / r_{i}=1$ with no modeling errors.

Similarly, Fig. 12 illustrates the impact of inaccuracies in the calibration of the COM position on the steady-state tracking performance. We focus our attention on the horizontal position error for the COM, denoted by $y_{C O M}^{\mathcal{B}}$, and defined as the horizontal position of the actual body center of mass in the body frame. We found this horizontal COM error to have significantly more effect on the stability and performance of pronking compared to vertical position errors. Beyond a certain discrepancy, particularly in the backwards direction, the pronking controller becomes increasingly unreliable and does not converge to a limit cycle. Fortunately, the reliable range of $-0.05<y_{\text {COM }}^{\mathcal{B}}<0.4$ $\left(-0.01 \mathrm{~m}<y_{C O M}^{\mathcal{B}}<0.07 \mathrm{~m}\right.$ for RHex with $\left.l_{0}=17.5 \mathrm{~cm}\right)$ is
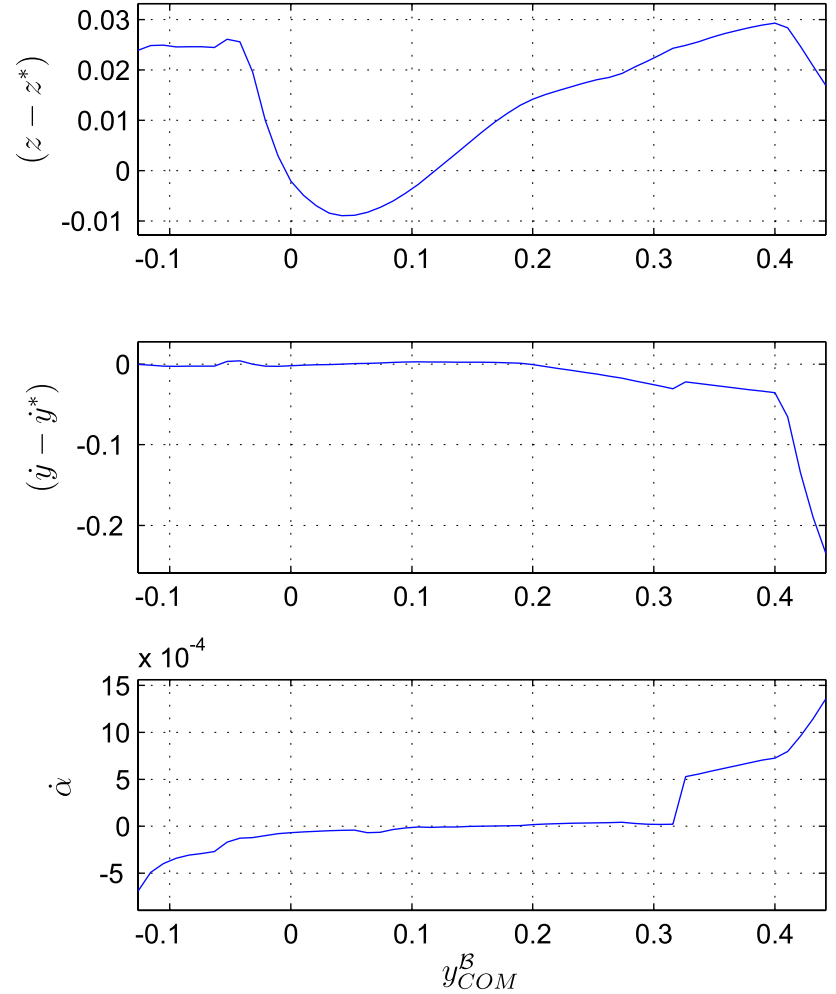

Fig. 12 Sensitivity of steady-state tracking performance of the pronking controller with respect to a miscalibrated horizontal COM position. $y_{C O M}^{\mathcal{B}}$ denotes the horizontal position of the actual COM in the body frame, with positive values corresponding to a front-heavy robot

reasonably large and practically feasible. It is usually practical to obtain center of mass estimates within such centimeter scale ranges with modern solid modeling tools even though unpredictable payloads may be more problematic. Nevertheless, in this range, the pitch velocity at apex remains largely unaffected by the errors, whereas the height parameter suffers the most. Most interestingly, however, the results show that when the actual body center of mass is ahead of the geometric center of the robot, there is a notable increase in the tracking performance. This effect is a natural result of the fact that when the body COM is shifted forward, the posi- 
tive pitch torque provided by gravity helps balance the effect of leg torques in the opposite direction.

Another possible source of model uncertainty arises if there is toe slippage, violating of the fixed toe assumption within the Slimpod model. However, the embedding controller of Sect. 4.2 is based on instantaneous inversion of the second order system dynamics and does not inherently rely on past states. Consequently, if there is a sufficiently accurate sensory suite on a physical robot implementation that can track foot contact locations relative to the body position, the embedding controller can be informed of associated kinematic changes and minimize the impact of foot slippage on controller performance. Since our focus in this paper is on the ideal performance of the embedding controller and insights that may be gained from its study, we leave issues related to the realization of such a sensory suite, which naturally entails numerous challenges, outside the scope of this paper.

\subsubsection{Sensitivity to discrete control and sensor noise}

Our final set of simulations investigate the performance of our pronking controller under substantial noise conditions. In contrast to the simulations of preceding sections, all of which were obtained using simultaneous integration of model and controller dynamics, we will now discretize our controller actions and apply piecewise constant torque commands at a frequency of $1 \mathrm{KHz}$. This is a much more realistic scenario since any physical robotic platform will have similar constraints, having to perform closed loop control digitally at a limited frequency.

In addition to this "discretization noise", we also separately add zero-mean, white Gaussian noise with increasing amounts of standard deviation to our force and state measurement readings in an attempt to characterize the sensitivity of our controller with respect to these sensory inputs. Since our aim is controlling the apex variables, we investigate the effect of the noise measurements on the apex height and apex velocity.

We summarize the effects of sensory noise on pronking performance through the relation of the standard deviation in the steady-state tracking errors (taking into account the last 10 apex states for each run) to the standard deviation of the sensory noise. More specifically, we ran simulations using different noise conditions with standard deviation $\sigma_{\text {noise }}$ to determine the following relations

$\sigma_{z_{a}}=\beta_{z_{a}} \sigma_{\text {noise }}+\gamma_{z_{a}}$,

$\sigma_{\dot{y}_{a}}=\beta_{\dot{y}_{a}} \sigma_{\text {noise }}+\gamma_{\dot{y}_{a}}$,

where the affine parameters $\beta_{z_{a}}, \gamma_{z_{a}}, \beta_{\dot{y}_{a}}, \gamma_{\dot{y}_{a}}$ were determined using linear regression. Table 5 summarizes our results where each row includes the fitted parameters for noise
Table 5 Sensitivity of steady-state tracking errors to sensory noise on different state variables. $\beta$ and $\gamma$ are slopes and offsets of a linear relation between the standard deviation of the steady state error and the standard deviation of the noise

\begin{tabular}{lllllr}
\hline State variable & \multicolumn{2}{l}{ Apex height } & & \multicolumn{2}{c}{ Apex speed } \\
\cline { 2 - 3 } & $\beta_{z_{a}}$ & $\gamma_{z_{a}}$ & & $\beta_{\dot{y}_{a}}$ & \multicolumn{1}{c}{$\gamma_{\dot{y}_{a}}$} \\
\hline Horizontal position & 0.189 & 0.0038 & & 0.954 & 0.0047 \\
Vertical position & 0.223 & 0.0063 & & 2.067 & -0.0016 \\
Horizontal speed & 0.424 & 0.0011 & 1.421 & -0.0010 \\
Vertical speed & 0.288 & 0.0017 & 1.151 & 0.0005 \\
Pitch angle & 0.171 & 0.0063 & 0.940 & 0.0051 \\
Pitch rate & 0.700 & 0.0008 & 1.411 & 0.0001 \\
Force & 0.028 & 0.0008 & 0.078 & -0.0011 \\
\hline
\end{tabular}

injected into a single specific sensory variable. The analysis in this section is intended to help identify the relative importance of sensing on different components of the robot state with respect to their impact on controller performance.

Somewhat surprisingly, our results show that force variables do not have a critical impact on controller performance which is encouraging since it is very hard to reliably implement accurate force measurements on dynamic, autonomous hexapods such as RHex. However, vertical position, and both velocity coordinates seem to have substantial impact on particularly the apex speed tracking performance. This is not entirely surprising since these state variables directly effect the total energy in the system and hence influence the performance of the embedding controller. These position and velocity state variables are among the hardest quantities to measure on autonomous legged robots, but if for a known ground profile and well instrumented legs, accurate and high bandwidth estimation of these state components may be possible (Lin 2005). Since the vertical position seems to be the most critical state component, additional sensory readings such as laser range sensors monitoring distance to the ground may be used for better estimates.

The pitch angle and rate components are the least problematic from a practical point of view since even solid-state inertial measurement units are capable of accurate estimation of pitch and roll degrees of freedom particularly when a good motion model is available for filtering. The relatively high dependence of tracking performance on these variables is not entirely surprising since they have significant impact on the kinematics of front and back legs, introducing errors in the embedding accuracy. Finally, the offset terms $\gamma_{z_{a}}$ and $\gamma_{\dot{y}_{a}}$ simply provide a sanity check and show that our simulations have indeed converged to a limit cycle, with very small variation in the last 10 apex states when no state measurement noise is present. The variations that are observed are a consequence of the $1 \mathrm{KHz}$ frequency we impose on the controller actions, which are not necessarily phase-aligned with apex events. 
Overall, our results show that state elements that critically contribute to controller stability and performance are also those that can practically be estimated in a physical robot platform. There is of course still substantial work to be done to achieve the required sensory accuracy on an autonomous legged platform such as RHex, but our results in this section can be used to identify the relative level of accuracy required for different components of the robot state.

\section{Conclusion}

In this paper, we presented a new method for controlling dynamic locomotory behaviors based on the identification of a low dimensional template system that accurately captures task dynamics, often motivated by observations of similar behaviors in nature, and the embedding of this template into a particular robotic morphology. This method not only simplifies the control problem by dividing it into two separate, smaller and easier to solve pieces, but also makes high level control of the resulting behavior much simpler due to the task-specific interface entailed by the template model. This decomposition is similar in spirit to how the loose coupling between forward speed and hopping height control for the basic SLIP model was exploited by Raibert's controllers. However, the hexapedal morphology does not feature such passively decoupled coordinates, which is why active feedback and our embedding methodology is needed to yield a similar structure.

We illustrated the utility of this methodology on the problem of achieving stable and controllable hexapedal pronking, which has been very difficult to achieve in the absence of radial leg actuation. To this end, we adopted the SpringLoaded Inverted Pendulum (SLIP) template, a simple, lowdimensional model that has long been established as the best descriptive dynamical model for running behaviors. Using a deadbeat controller acting on the SLIP template together with its embedding within a planar hexapod model as a virtual leg, we have been able to achieve robust and stable pronking, whose forward speed and hopping height can be explicitly regulated. Finally, in order to establish practical feasibility of our controller, we investigated in simulation, the sensitivity of its steady-state performance to inaccuracies in the calibration of model parameters, a realistic actuation model with piecewise constant torque outputs and varying levels of sensor noise. Despite our reliance on simulation studies due to present limitations of our experimental platform, RHex, with respect to sufficiently accurate and high-bandwidth sensory information, our results show that the pronking controller is sufficiently robust to support a physical implementation.

Our intent in the near future is to implement this controller in a planarized hexapod wherein accurate state feedback and hence a direct implementation of the controller would be possible. However, our long term goal is the identification of critical aspects of the control actions taken by this high-bandwidth controller and design a corresponding open-loop controller (with possibly limited feedback at each stride) that inherits the stability and gait-level controllability properties of the feedback controller. We believe that such a quasi-open-loop controller informed by observations on a successful feedback controller will be much more practical and robust for a legged robot in the field, where accurate, high-bandwidth state estimation will be extremely difficult, if not impossible to realize.

Acknowledgements This work was partially supported by TUBITAK, the Scientific and Technical Research Council of Turkey through M. Mert Ankaralı's scholarship and TUBITAK Project 109E032.

\section{References}

Allen, T., Quinn, R., Bachmann, R., \& Ritzmann, R. (2003). Abstracted biological principles applied with reduced actuation improve mobility of legged vehicles. In Proceedings. 2003 IEEE/RSJ international conference on intelligent robots and systems, IROS 2003, (vol. 2, pp. 1370-1375).

Altendorfer, R. E. (2000). Evidence for spring loaded inverted pendulum running in a hexapod robot. In D. Rus \& S. Singh (Eds.), Lecture Notes in Control and Information Sciences: Vol. 5. Experimental Robotics VII (pp. 291-302). Berlin: Springer.

Altendorfer, R., Koditschek, D. E., \& Holmes, P. (2004). Stability analysis of legged locomotion models by symmetry-factored return maps. The International Journal of Robotics Research, 23(10-11), 979-999.

Ankarali, M. M., Arslan, O., \& Saranli, U. (2009). An analytical solution to the stance dynamics of passive spring-loaded inverted pendulum with damping. In 12th international conference on climbing and walking robots and the support technologies for mobile machines (CLAWAR'09), Istanbul, Turkey.

Arslan, O., Saranli, U., \& Morgul, O. (2009). An approximate stance map of the spring mass hopper with gravity correction for nonsymmetric locomotions. In Proceedings of the international conference on robotics and automation, Kobe, Japan.

Berkemeier, M., \& Sukthankar, P. (2005). Self-organizing running in a quadruped robot model. In Proceedings of the international conference on robotics and automation (pp. 4108-4113).

Blickhan, R., \& Full, R. J. (1993). Similarity in multilegged locomotion: bouncing like a monopode. Journal of Comparative Physiology A: Neuroethology, Sensory, Neural, and Behavioral Physiology, 173(5), 509-517.

Caro, T. M. (1994). Ungulate antipredator behaviour: preliminary and comparative data from African bovids. Behavior, 128(3-4), 189228.

Carver, S. Control of a spring-mass Hopper. Ph.D., Cornell University, January 2003.

Chatzakos, P., \& Papadopoulos, E. (2007). Parametric analysis and design guidelines for a quadruped bounding robot. In Proceedings of the med. conference on control and automation (pp. 1-6).

Chatzakos, P., \& Papadopoulos, E. (2009). Bio-inspired design of electrically-driven bounding quadrupeds via parametric analysis. Mechanism and Machine Theory, 44(3), 559-579. Special Issue on Bio-Inspired Mechanism Engineering.

Chatzkos, P., \& Papadopoulos, E. (2009). A parametric study on the rolling motion of dynamically running quadrupeds during pronking. In Proceedings of the med. conference on control and automation, Thessaloniki, Greece, June 2009 (pp. 754-759). 
Chevallereau, C., Grizzle, J. W., \& Shih, C.-L. (2009). Asymptotically stable walking of a five-link underactuated $3 \mathrm{~d}$ bipedal robot. IEEE Transactions on Robotics, 25(1), 37-50.

Duysens, J., \& de Crommert, H. W. A. A. V. (1998). Neural control of locomotion; part 1: the central pattern generator from cats to humans. Gait \& Posture, 7(2), 131-141.

FitzGibbon, C. D., \& Fanshawe, J. H. (1988). Stotting in Thomson's gazelles: an honest signal of condition. Behavioral Ecology and Sociobiology, 23(2), 69-74.

Full, R. J., \& Koditschek, D. E. (1999). Templates and anchors: neuromechanical hypotheses of legged locomotion. Journal of Experimental Biology, 202, 3325-3332.

Geyer, H., Seyfarth, A., \& Blickhan, R. (2005). Spring-mass running: simple approximate solution and application to gait stability. Journal of Theoretical Biology, 232(3), 315-328.

Greenfield, A., Saranli, U., \& Rizzi, A. A. (2005). Solving models of controlled dynamic planar rigid-body systems with frictional contact. The International Journal of Robotics Research, 24(11), 911-931.

Gregorio, P., Ahmadi, M., \& Buehler, M. (1997). Design, control, and energetics of an electrically actuated legged robot. Transactions on Systems, Man, and Cybernetics, 27(4), 626-634.

Klavins, E., Komsuoglu, H., Full, R. J., \& Koditschek, D. E. (2002). The role of reflexes versus central pattern generators in dynamical legged locomotion. In Neurotechnology for biomimetic robots (pp. 351-382). Boston: MIT Press.

Kopell, N. (2000). We got rhythm: dynamical systems of the nervous system. American Mathematical Society, 47(1), 6-16.

Kuo, A. D. (2002). The relative roles of feedforward and feedback in the control of rhythmic movements. Motor Control, 6(2), 129145 .

Lin, P.-C. (2005). Proprioceptive sensing for a legged robot. Ph.D., The University of Michigan, Ann Arbor, MI.

McMordie, D. (2002). Towards pronking with a hexapod robot. Master's thesis, McGill University.

McMordie, D., \& Buehler, M. (2001). Towards pronking with a hexapod robot. In 4th international conference on climbing and walking robots, Karlsruhe, Germany.

Poulakakis, I., Smith, J. A., \& Buehler, M. (2005). Modeling and experiments of untethered quadrupedal running with a bounding gait: the scout II robot. The International Journal of Robotics Research, 24(4), 239-256.

Raibert, M. (1986). MIT Press series in artificial intelligence. Legged robots that balance. Boston: MIT Press.

Raibert, M. H. (1990). Trotting, pacing and bounding by a quadruped robot. Journal of Biomechanics, 23(1), 79-98.

Saranli, U. (2000). SimSect hybrid dynamical simulation environment. (Technical report CSE-TR-436-00). UM, Ann Arbor, MI.

Saranli, U. (2002). Dynamic locomotion with a hexapod robot. Ph.D. thesis, The University of Michigan, Ann Arbor, MI.

Saranli, U., Buehler, M., \& Koditschek, D. E. (2001). RHex: a simple and highly mobile robot. The International Journal of Robotics Research, 20(7), 616-631.

Saranli, U., \& Koditschek, D. E. (2003). Template based control of hexapedal running. In Proceedings of the IEEE international conference on robotics and automation, Taipei, Taiwan (vol. 1, pp. 1374-1379).

Saranli, U., Rizzi, A. A., \& Koditschek, D. E. (2004). Model-based dynamic self-righting maneuvers for a hexapedal robot. The International Journal of Robotics Research, 23(9), 903-918.
Saranli, U., Schwind, W. J., \& Koditschek, D. E. (1998). Toward the control of a multi-jointed, monoped runner. In Proceedings of the IEEE international conference on robotics and automation, New York (vol. 3, pp. 2676-2682).

Sato, A., \& Buehler, M. (2004). A planar hopping robot with one actuator: design, simulation, and experimental results. In Proceedings of the international conference on intelligent robots and systems (vol. 4, pp. 3540-3545).

Schwind, W. J. (1998). Spring loaded inverted pendulum running: a plant model. Ph.D., University of Michigan.

Westervelt, E. R., Grizzle, J. W., Chevallerau, C., Choi, J.-H., \& Morris, B. (2007). Feedback control of dynamic bipedal robot locomotion. London: Taylor and Francis.

Zeglin, G. (1999). The bow leg hopping robot. Doctoral thesis in robotics, Carnegie Mellon University.

Zou, H., \& Schmiedeler, J. (2006). The effect of asymmetrical bodymass distribution on the stability and dynamics of quadruped bounding. IEEE Transactions on Robotics, 22(4), 711-723.

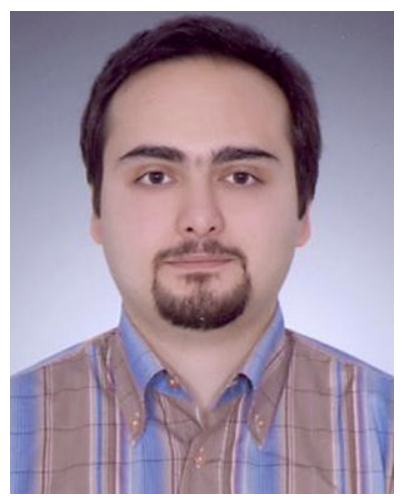

M. Mert Ankaralı received his B.Sc. degree in Mechanical Engineering and minor certificate in Mechatronics from the Middle East Technical University, Turkey in 2007, and 2008, respectively. He received his M.Sc. degree from the Electrical \& Electronics Engineering Department of Middle East Technical University, Turkey in 2010. He is currently a doctoral student in the Dept. of Mechanical Engineering, Johns Hopkins University. His research interests include legged locomotion, bio-inspired robotics, and physically realistic simulation systems.

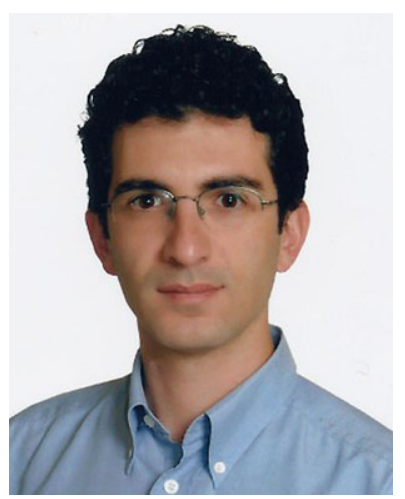

Uluc Saranlı is currently an Assistant Professor in the Dept. of Computer Engineering, Bilkent University, Turkey. He received his B.Sc. degree from the Dept. of Electrical and Electronics Engineering, Middle East Technical University, Turkey in 1996. He subsequently received his M.Sc. and Ph.D degrees from the Computer Science Department in the University of Michigan, Ann Arbor in 1998 and 2002, respectively. He was a Postdoctoral Fellow in the Robotics Institute, Carnegie Mellon University until his joining of Bilkent University in 2005. His research interests focus on the analysis and control of legged locomotion, design of autonomous robots, the use of logical formalisms for robotic autonomy and planning as well as embedded systems and control. 